\title{
The Death of a Baby from the Congenital Anomalies of the Urinary Tract
}

\author{
Gashi AM*, Sopa G, Kadiri I, Balaj M and Pupa P
}

Department of Obstetrics and Gynecology, University Clinical Centre of Kosovo, Pristine

*Corresponding author: Gashi AM, Department of Obstetrics and Gynecology, University Clinical Centre of Kosovo, Pristine, Tel: +37744266902, E-mail: astritgashi772@gmail.com

Citation: Gashi AM, Sopa G, Kadiri I, Balaj M, Pupa P (2018) The Death of a Baby from the Congenital Anomalies of the Urinary Tract. J Gynecol Res 4(1): 104. doi: 10.15744/2454-3284.4.104

Received Date: February 13, 2018 Accepted Date: April 10, 2018 Published Date: April 12, 2018

Keywords: Teenage girls; Pregnancy; The birth rate; Complications

A 36-year-old woman pregnant, G2 P1, presented at 27 weeks of gestation after two previous visits elsewhere, as an outpatient in a gynecological clinic. An ultrasound examination revealed bilateral hydronephrosis. Also, ureteral dilation and bladder overdistension was present (Figure 1, 2 and 3). We evaluated that the cause was a urinary tract obstruction. Specifically, we are dealing with posterior urethral valves. The anteroposterior diameter of the pelvis on a transverse view of the abdomen was 6 mm. The amniotic fluid index (AFI) was $3 \mathrm{~cm}$, so, oligohydramnios. Fetal Biometry responds to the 27th week of gestation. The sex of the fetus was male. Following identification of urinary tract dilation, a detailed anatomic scan was performed to exclude the presence of associated extra-renal anomalies. After corticosteroid regimens, at week 30 of pregnancy, due to the fetal diagnosis previously determined and now anhydramnios, with the informed consent of both parents, are deciding to end a pregnancy with Caesarean section. On 17.07.2017, at 8:45 AM, the baby of the male sex is born, with body weight 1700 grams, and Apgar score was; 7 in the first minute, 8 in the fifth minute. Shortly after birth, because of the prematurity and the manifestation of the signs of Infant respiratory distress syndrome (IRDS), the baby is transferred to the Neonatal Intensive Care Unit. The treatment started according to the protocol with fluid, antibiotic therapy, and surfactant administration. Even despite therapy, the condition is constantly worsening the baby is intubated and passes on mechanical ventilation. The baby's condition continues to worsen even further until cardiac arrest, even despite reanimation measures, the baby died within a few hours.

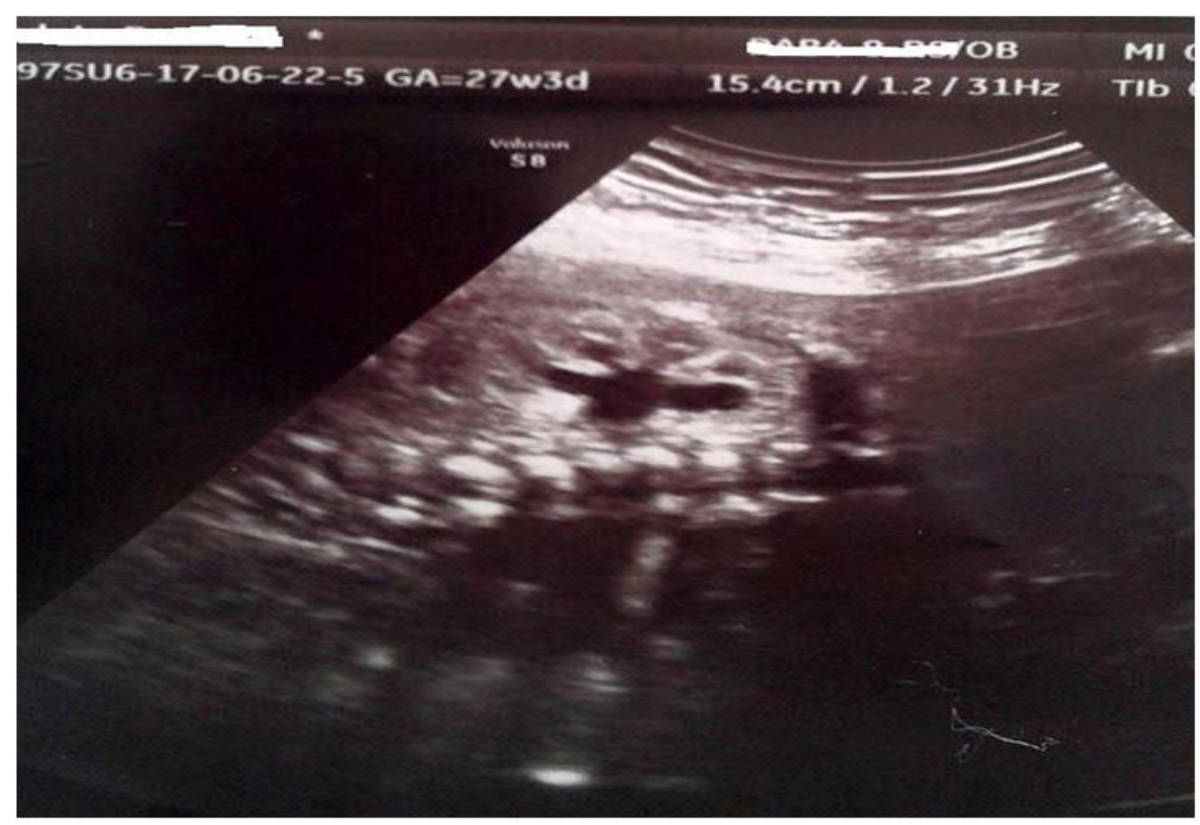

Figure 1: Coronal scan of a hydronephrotic fetal kidney in a 27 -week fetus, showing dilation of the pelvis, the calyces are visible but not significantly dilated 


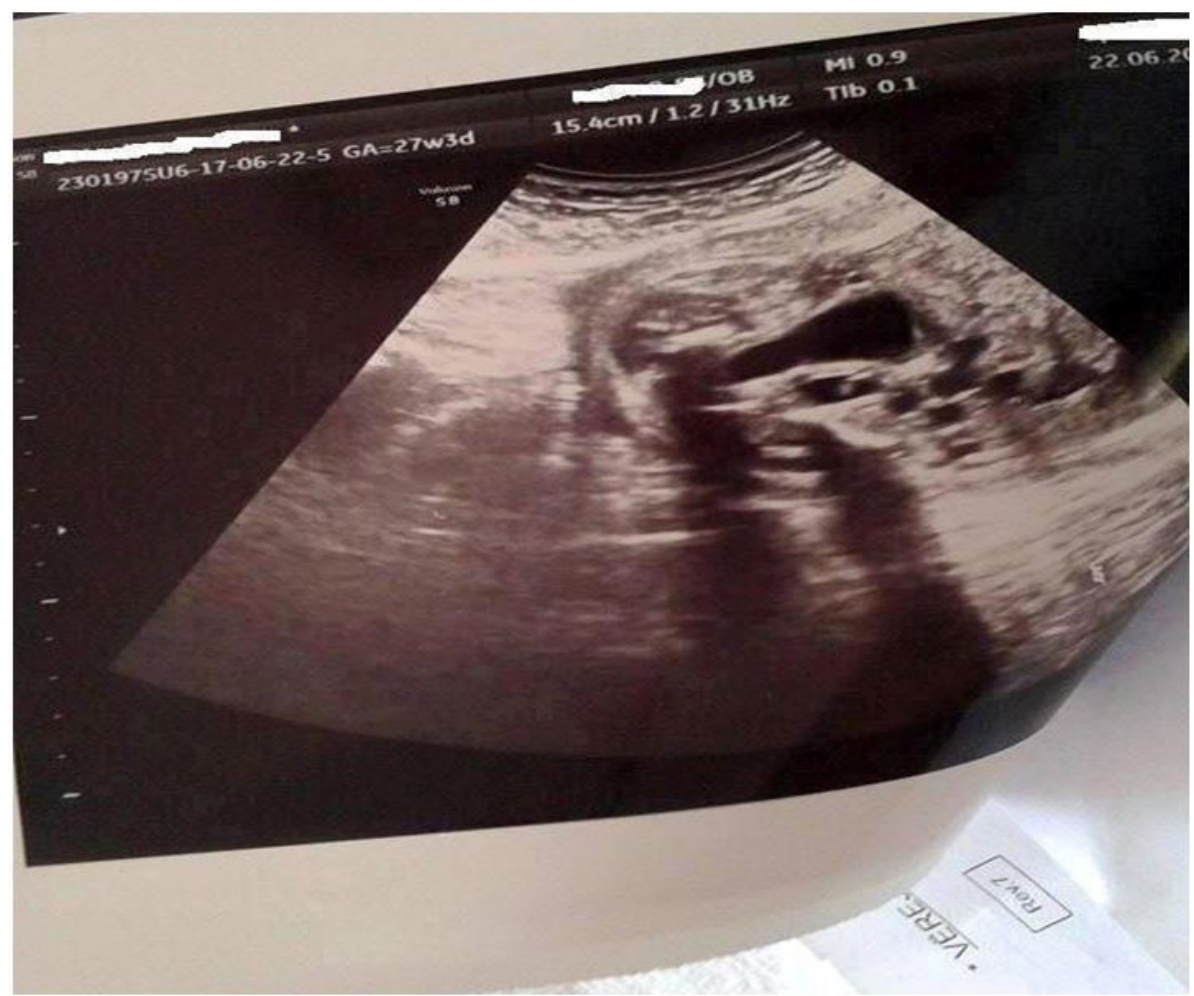

Figure 2: Hydronephrosis and ureteral dilation are present

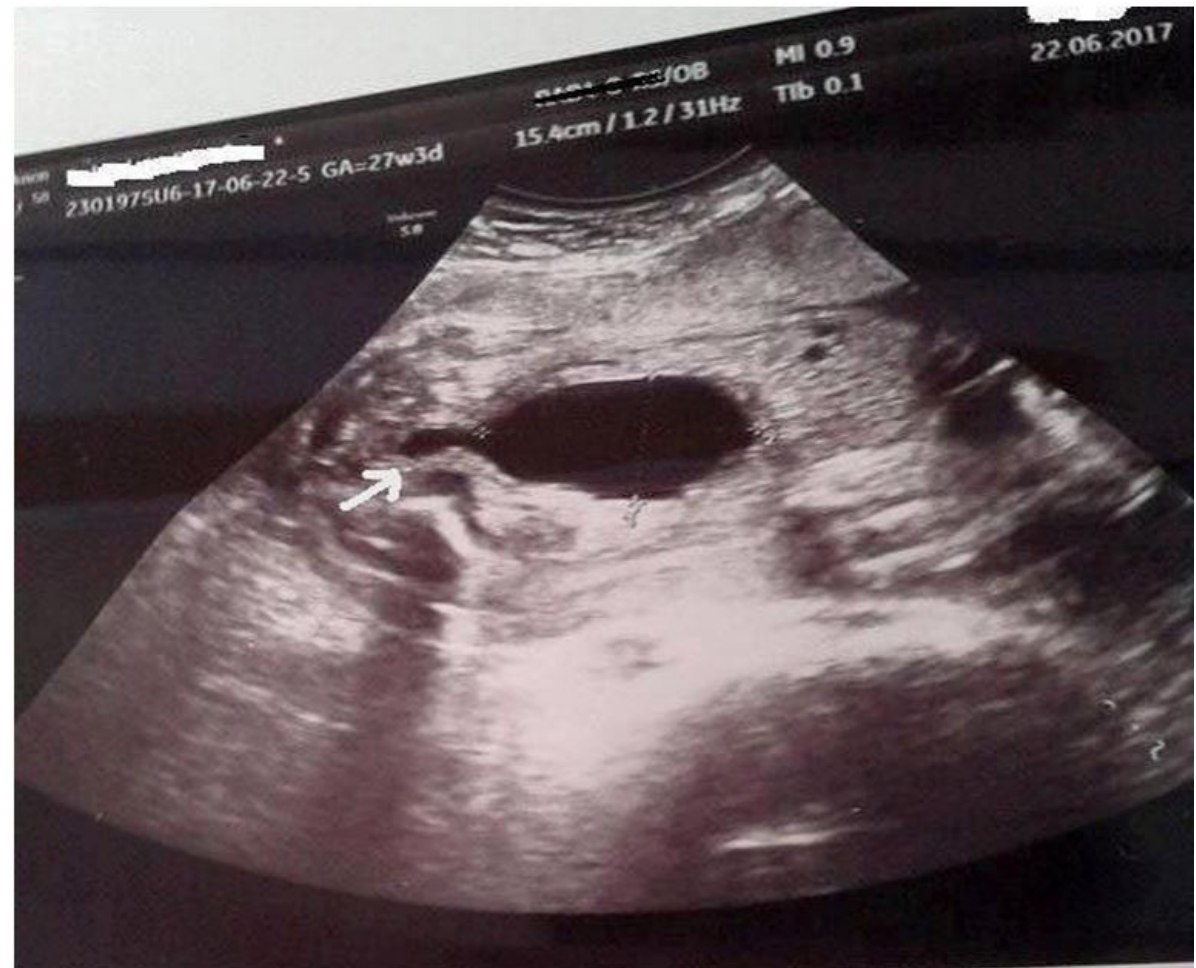

Figure 3: Posterior urethral valves. In a $27+3$ weeks fetus, a significant dilatation of the bladder and of the proximal part of the urethra (arrow) is clearly seen 


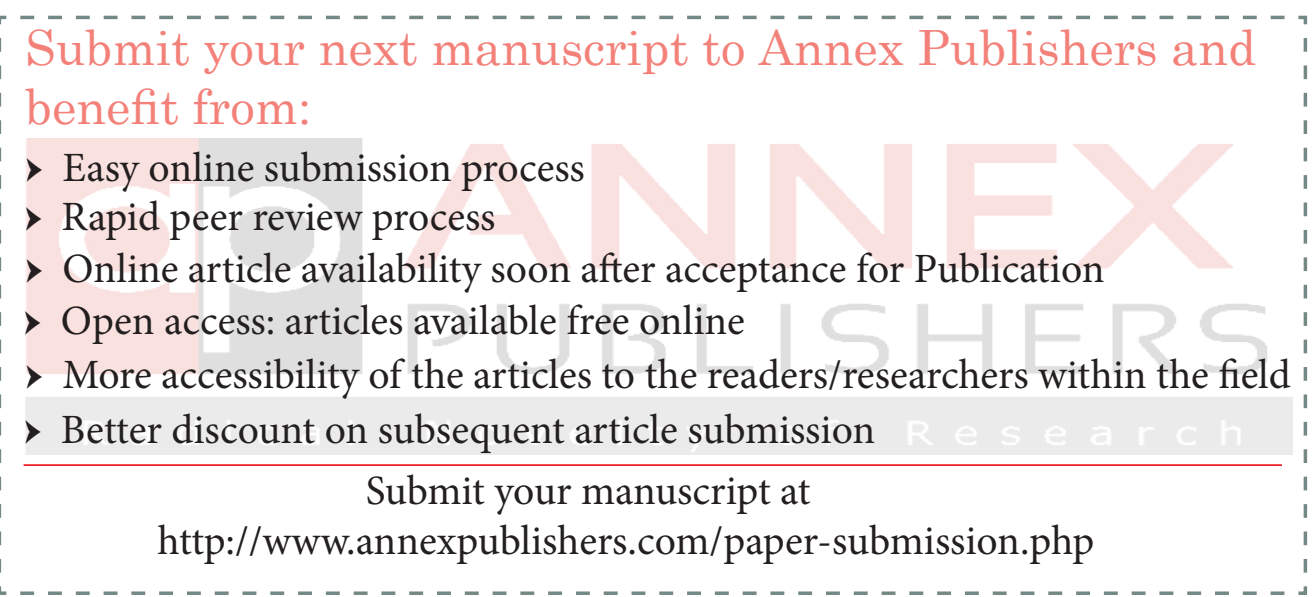

